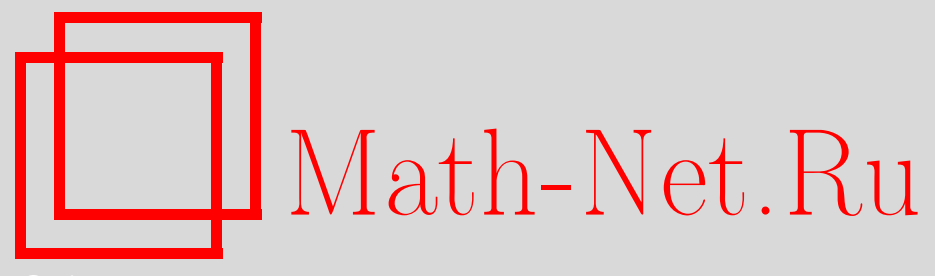

А. Р. Зайнуллов, Обратная задача для двумерного уравнения теплопроводности по отысканию начального распределения, Вестн. Сам. гос. техн. ун-та. Сер. Физ.-мат. науки, 2015, номер 4, 667679

DOI: https://doi.org/10.14498/vsgtu1451

Использование Общероссийского математического портала MathNet.Ru подразумевает, что вы прочитали и согласны с пользовательским соглашением

http://www.mathnet.ru/rus/agreement

Параметры загрузки:

IP: 54.237 .59 .107

26 апреля 2023 г., $17: 51: 22$

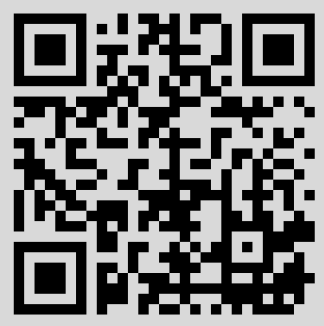


УДК 517.956.47

\title{
ОБРАТНАЯ ЗАДАЧА ДЛЯ ДВУМЕРНОГО УРАВНЕНИЯ ТЕПЛОПРОВОДНОСТИ ПО ОТЫСКАНИЮ НАЧАЛЬНОГО РАСПРЕДЕЛЕНИЯ
}

\section{A. Р. Зайнуллов}

Башкирский государственный университет, Стерлитамакский филиал,

Россия, 453103, Стерлитамак, проспект Ленина, 49

\begin{abstract}
Аннотация
На основании формулы решения первой начально-граничной задачи для неоднородного двумерного уравнения теплопроводности изучена обратная задача по отысканию начального распределения. В явном виде строится решение прямой начально-граничной задачи. Единственность решения прямой начально-граничной задачи доказана на основании свойства полноты системы собственных функций соответствующей однородной задачи Дирихле для оператора Лапласа. Доказана теорема существования решения прямой начально-граничной задачи. На основе решения этой задачи исследуется обратная задача, установлен критерий единственности решения обратной задачи. Существование решения обратной задачи эквивалентно сведено к разрешимости интегрального уравнения Фредгольма первого рода.
\end{abstract}

Ключевые слова: уравнение теплопроводности, первая начально-граничная задача, обратная задача, спектральный метод, единственность, существование, интегральное уравнение.

doi: http://dx.doi.org/10.14498/vsgtu1451

\section{1. Введение}

Рассмотрим уравнение теплопроводности

$$
L u \equiv u_{t}-a^{2}\left(u_{x x}+u_{y y}\right)=F(x, y, t)
$$

в параллелепипеде

$$
D=\Pi \times(0, T), \quad \Pi=\{(x, y) \mid 0<x<l, 0<y<q\}
$$

и следующие задачи для него.

ПЕРВАЯ НАЧАЛЬНО-ГРАНИЧНАЯ ЗАДАЧА. Найтu в области $D$ функиию $u(x, y, t)$, удовлетворяющую следующим условиям:

$$
u(x, y, t) \in C(\bar{D}) \cap C_{x, y, t}^{2,2,1}(D) ;
$$

(C) 2015 Самарский государственный технический университет.

\section{Образец для цитирования}

З ай н у л л о в А. Р. Обратная задача для двумерного уравнения теплопроводности по отысканию начального распределения // Вестн. Сам. гос. техн. ун-та. Сер. Физ.-мат. науки, 2015. T. 19, № 4. C. 667-679. doi: 10.14498/vsgtu1451.

\section{Сведения об авторе}

Apmyp Рашитович Зайнуллов (arturzayn@mail.ru), аспирант, каф. математического анализа. 


$$
\begin{gathered}
L u \equiv F(x, y, t), \quad(x, y, t) \in D ; \\
u(0, y, t)=u(l, y, t)=0, \quad 0 \leqslant y \leqslant q, \quad 0 \leqslant t \leqslant T, \\
u(x, 0, t)=u(x, q, t)=0, \quad 0 \leqslant x \leqslant l, \quad 0 \leqslant t \leqslant T, \\
u(x, y, 0)=\varphi(x, y), \quad 0 \leqslant x \leqslant l, \quad 0 \leqslant y \leqslant q,
\end{gathered}
$$

где $\varphi(x, y)$ и $F(x, y, t)$ - заданнъе функиии.

ОБРАТНАЯ ЗАДАчА. Найти функиии $u(x, y, t) u \varphi(x, y)$, удовлетворяющие условиям (2)-(6) и, кроме того, дополнительному условию

$$
u\left(x_{0}, y_{0}, t\right)=h(t), \quad 0<t_{0} \leqslant t \leqslant t_{1} \leqslant T,
$$

где $\left(x_{0}, y_{0}\right)$ - заданная фиксированная точка прямоугольника $\Pi ; t_{0} u t_{1}-$ заданные действительные числа; $F(x, y, t), h(t)$ - заданные функиии.

Отметим, что указанная обратная задача изучена в [1, с. 119] для однородного одномерного уравнения теплопроводности с однородными граничными условиями второго рода и доказана теорема её единственности при $x_{0}=0$ и $x_{0}=l / \pi$.

В данной работе в явном виде строится решение прямой начально-граничной задачи (2)-(6) и на основе решения этой задачи исследуется обратная задача (2)-(7). В известных книгах по уравнениям математической физики [2-6] единственность решения начально-граничных задач доказывается методом интегралов энергии. Здесь, следуя [7, с. 111], [8], единственность решения задачи (2)-(6) доказана на основании свойства полноты системы собственных функций соответствующей однородной задачи Дирихле для оператора Лапласа. Также указаны достаточные условия относительно функций $\varphi(x, y)$ и $F(x, y, t)$, при которых решение поставленной задачи существует и оно определяется в виде суммы двойного ряда Фурье, так как в указанных книгах не приводятся обоснование сходимости ряда Фурье в классе функций (2).

На основании этих результатов установлен критерий единственности решения задачи $(2)-(7)$ для любой точки $\left(x_{0}, y_{0}\right)$ прямоугольника П при $l=q$.

\section{2. Единственность и существование прямой задачи}

Теорема 1. Если существует решение задачи (2)-(6), удовлетворяющее условиям

$$
\begin{aligned}
& \lim _{x \rightarrow 0+} u_{x} \sin \frac{\pi m x}{l}=\lim _{x \rightarrow l-} u_{x} \sin \frac{\pi m x}{l}=0, \\
& \lim _{y \rightarrow 0+} u_{y} \sin \frac{\pi n y}{l}=\lim _{y \rightarrow l-} u_{y} \sin \frac{\pi n y}{l}=0,
\end{aligned}
$$

то оно единственно.

Доказательств о. Пусть $\mu_{m n}^{2}$ - собственные значения и $X_{m n}(x, y)-$ соответствующие им собственные функции спектральной задачи:

$$
\begin{gathered}
v_{x x}+v_{y y}+\mu^{2} v=0, \quad(x, y) \in \Pi, \\
\left.v\right|_{x=0}=\left.v\right|_{x=l}=0,\left.\quad v\right|_{y=0}=\left.v\right|_{y=q}=0,
\end{gathered}
$$

которые определяются по формулам

$$
X_{m n}(x, y)=\frac{2}{l} \sin \mu_{m} x \sin \mu_{n} y,
$$




$$
\mu_{m n}^{2}=\mu_{m}^{2}+\mu_{n}^{2}, \mu_{m}=\frac{\pi m}{l}, \quad \mu_{n}=\frac{\pi n}{l}, \quad m, n=1,2, \ldots
$$

Наряду с этой системой рассмотрим систему

$$
Z_{m n}(x, y)=\frac{1}{2}\left[X_{m n}(x, y)+X_{n m}(x, y)\right]
$$

которая обладает свойством симметрии

$$
Z_{m n}(x, y)=Z_{n m}(x, y)
$$

Система (11) является решением спектральной задачи (9) и (10), обладает свойствами ортогональности и полноты в пространстве $L_{2}(\Pi)$.

Рассмотрим интеграл

$$
v_{m n}^{\varepsilon, \delta}(t)=\int_{\varepsilon}^{l-\varepsilon} \int_{\delta}^{l-\delta} u(x, y, t) Z_{m n}(x, y) d x d y
$$

где $\varepsilon, \delta$ - достаточно малые положительные числа.

Дифференцируя равенство (12) при $t \in(0, T)$ и учитывая уравнение $(1)$, получим

$$
\begin{gathered}
v_{m n}^{\prime}(t)=\int_{\varepsilon}^{l-\varepsilon} \int_{\delta}^{l-\delta} u_{t}(x, y, t) Z_{m n}(x, y) d x d y= \\
=\int_{\varepsilon}^{l-\varepsilon} \int_{\delta}^{l-\delta}\left(a^{2} \Delta u+F(x, y, t)\right) Z_{m n}(x, y) d x d y= \\
\left.=a^{2} \int_{\varepsilon}^{l-\varepsilon} \int_{\delta}^{l-\delta} u_{x x} Z_{m n} x, y\right) d x d y+a^{2} \int_{\varepsilon}^{l-\varepsilon} \int_{\delta}^{l-\delta} u_{y y} Z_{m n}(x, y) d x d y+ \\
\quad+\int_{\varepsilon}^{l-\varepsilon} \int_{\delta}^{l-\delta} F(x, y, t) Z_{m n}(x, y) d x d y= \\
=a^{2}\left(I_{1}+I_{2}\right)+F_{m n}(t)
\end{gathered}
$$

где

$$
F_{m n}=\int_{\varepsilon}^{l-\varepsilon} \int_{\delta}^{l-\delta} F(x, y, t) Z_{m n}(x, y) d x d y .
$$

С учетом граничных условий (4), (5), $X(0)=X(l)=0, Y(0)=Y(q)=0$ проинтегрируем по частям интегралы $I_{1}$ и $I_{2}$ в области П:

$$
\begin{aligned}
I_{1}=\int_{\delta}^{l-\delta} d y \int_{\varepsilon}^{l-\varepsilon} u_{x x} Z_{m n}(x, y) d x= & \\
= & \int_{\delta}^{l-\delta} d y\left(\left.u_{x} Z_{m n}(x, y)\right|_{\varepsilon} ^{l-\varepsilon}-\int_{\varepsilon}^{l-\varepsilon} u_{x}\left(Z_{m n}(x, y)\right)_{x}^{\prime} d x\right)= \\
= & \int_{\delta}^{l-\delta} d y\left(\left.u_{x} Z_{m n}(x, y)\right|_{\varepsilon} ^{l-\varepsilon}-\left.u(x, y, t)\left(Z_{m n}(x, y)\right)^{\prime}\right|_{\varepsilon} ^{l-\varepsilon}+\right. \\
& \left.+\int_{\varepsilon}^{l-\varepsilon} u(x, y, t)\left(Z_{m n}(x, y)\right)_{x x}^{\prime \prime} d x\right)
\end{aligned}
$$




$$
\begin{aligned}
I_{2}=\int_{\varepsilon}^{l-\varepsilon} & d x \int_{\delta}^{l-\delta} u_{y y} Z_{m n}(x, y) d y= \\
= & \int_{\varepsilon}^{l-\varepsilon} d x\left(\left.u_{y} Z_{m n}(x, y)\right|_{\delta} ^{l-\delta}-\int_{\delta}^{l-\delta} u_{y}\left(Z_{m n}(x, y)\right)_{y}^{\prime} d y\right)= \\
= & \int_{\varepsilon}^{l-\varepsilon} d x\left(\left.u_{y} Z_{m n}(x, y)\right|_{\delta} ^{l-\delta}-\left.u(x, y, t)\left(Z_{m n}(x, y)\right)_{y}^{\prime}\right|_{\delta} ^{l-\delta}+\right. \\
& \left.+\int_{\delta}^{l-\delta} u(x, y, t)\left(Z_{m n}(x, y)\right)_{y y}^{\prime \prime} d y\right) .
\end{aligned}
$$

Из (13) с учетом полученных значений интегралов $I_{1}$ и $I_{2}$ при $\varepsilon \rightarrow 0$, $\delta \rightarrow 0$, следует

$$
\begin{gathered}
v^{\prime}(t)=a^{2} \iint_{\Pi} u(x, y, t)\left(\left(Z_{m n}(x, y)\right)_{x x}^{\prime \prime}+\left(Z_{m n}(x, y)\right)_{y y}^{\prime \prime}\right) d x d y+F_{m n}(t)= \\
=-\mu_{m n}^{2} a^{2} \iint_{\Pi} u(x, y, t) Z_{m n}(x, y) d x d y+F_{m n}(t)= \\
=-\mu_{m n}^{2} a^{2} v_{m n}(t)+F_{m n}(t)
\end{gathered}
$$

откуда

$$
v_{m n}^{\prime}(t)+\mu_{m n}^{2} a^{2} v_{m n}(t)=F_{m n}(t) .
$$

Общее решение уравнения (15) определяется по формуле

$$
v_{m n}(t)=\int_{0}^{t} F_{m n}(s) e^{-\mu_{m n}^{2} a^{2}(t-s)} d s+C_{m n} e^{-\mu_{m n}^{2} a^{2} t},
$$

где $C_{m n}$ - произвольные постоянные. Для определения их воспользуемся граничным условием (6) и формулой (12):

$$
v_{m n}(0)=\iint_{\Pi} u(x, y, 0) Z_{m n}(x, y) d x d y=\iint_{\Pi} \varphi(x, y) Z_{m n}(x, y) d x d y=\varphi_{m n} .
$$

Удовлетворяя (16) начальному условию $(17)$, найдем $C_{m n}=\varphi_{m n}$ и

$$
v_{m n}(t)=\varphi_{m n} e^{-\mu_{m n}^{2} a^{2} t}+\int_{0}^{t} F_{m n}(s) e^{-\mu_{m n}^{2} a^{2}(t-s)} d s .
$$

Отсюда следует единственность решения задачи (2)-(6), так как если положить $\varphi(x, y) \equiv 0, F(x, y, t) \equiv 0$, то $\varphi_{m n} \equiv 0, F_{m n}(s) \equiv 0$, и из (18) получим $v_{m n}(t)=0$, что на основании (12) равносильно равенству

$$
\iint_{\Pi} u(x, y, t) Z_{m n}(x, y) d x d y=0
$$

и в силу полноты системы $Z_{m n}(x, y)$ в пространстве $L_{2}(\Pi)$ функция $u(x, y, t)=0$ почти всюду в П и при любом $t \in[0, T]$. Тем самым единственность решения задачи (2)-(6) доказана. 
Tеорема 2. Ecлu $\varphi(x, y) \in C^{4}(\bar{\Pi}), F(x, y, t) \in C_{x, y}^{2,2}(\bar{\Pi}) \cap C_{t}(0, T) u$

$$
\begin{gathered}
\varphi(0, y)=\varphi(l, y)=\varphi(x, 0)=\varphi(x, l)=0 \\
\varphi_{x}^{\prime}(0, y)=\varphi_{x}^{\prime}(l, y)=\varphi_{x}^{\prime}(x, 0)=\varphi_{x}^{\prime}(x, l)=0, \\
\varphi_{y}^{\prime}(0, y)=\varphi_{y}^{\prime}(l, y)=\varphi_{y}^{\prime}(x, 0)=\varphi_{y}^{\prime}(x, l)=0, \\
F(0, y, t)=F(l, y, t)=F(x, 0, t)=F(x, l, t)=0, \\
F_{x}^{\prime}(0, y, t)=F_{x}^{\prime}(l, y, t)=F_{x}^{\prime}(x, 0, t)=F_{x}^{\prime}(x, l, t)=0, \\
F_{y}^{\prime}(0, y, t)=F_{y}^{\prime}(l, y, t)=F_{y}^{\prime}(x, 0, t)=F_{y}^{\prime}(x, l, t)=0
\end{gathered}
$$

для всех $(x, y, t) \in \bar{D}$, то существует решение задачи (2)-(6) и оно представимо в виде суммы ряда

$$
u(x, y, t)=\sum_{m, n=1}^{\infty}\left[\varphi_{m n} e^{-\mu_{m n}^{2} a^{2} t}+\int_{0}^{t} F_{m n}(s) e^{-\mu_{m n}^{2} a^{2}(t-s)} d s\right] Z_{m n}(x, y)
$$

с коэфбициентами, которые определяются по бормулам (17) и (14).

Доказ а тель ст в о. Решение задачи (2)-(6) выше формально построено в виде суммы ряда $(21)$, где коэффициенты $\varphi_{m n}, F_{m n}(t)$ определяются по формулам (17) и (14). Если ряд (21) равномерно сходится на $\bar{D}$, так же как ряды, полученные из него почленным дифференцированием один раз по переменной $t$ и по два раза по $x, y$, то его сумма будет удовлетворять в области $D$ условиям (2)-(6).

Предварительно проинтегрируем по частям интегралы $(17),(14)$ два раза по $x$ и два раза $y$ и, учитывая (19), (20), получим

$$
\begin{aligned}
\varphi_{m n}=\iint_{\Pi} \varphi(x, y) Z_{m n}(x, y) d x d y= \\
=\frac{1}{l} \int_{0}^{l} \sin \mu_{n} y d y \int_{0}^{l} \varphi(x, y) \sin \mu_{m} x d x+ \\
\quad+\frac{1}{l} \int_{0}^{l} \sin \mu_{m} y d y \int_{0}^{l} \varphi(x, y) \sin \mu_{n} x d x= \\
=-\frac{1}{\mu_{m}^{2}} \frac{1}{l} \int_{0}^{l} \sin \mu_{n} y d y \int_{0}^{l} \varphi_{x x}^{\prime \prime} \sin \mu_{m} x d x- \\
=-\frac{1}{\mu_{m}^{2}} \frac{1}{l} \int_{0}^{l} \frac{1}{\mu_{n}^{2}} \int_{0}^{l} \sin \mu_{m} x d x \int_{0}^{l} \varphi_{x x}^{\prime \prime} \sin \mu_{n} y d y- \\
\quad-\frac{1}{\mu_{n}^{2}} \frac{1}{l} \int_{0}^{l} \sin \mu_{n} x d x \int_{0}^{l} \varphi_{x x}^{\prime \prime} \sin \mu_{n} x d x= \\
=\frac{1}{\mu_{m}^{2} \mu_{n}^{2}} \frac{1}{l} \int_{0}^{l} \sin \mu_{m} y d y= \\
+\frac{1}{\mu_{m}^{2} \mu_{n}^{2}} \frac{1}{l} \int_{0}^{l} \sin \mu_{n} x d x \int_{0}^{l} \varphi_{x y}^{2,2} \sin \mu_{m} y d y=
\end{aligned}
$$




$$
\begin{gathered}
=\frac{1}{\mu_{m}^{2} \mu_{n}^{2}} \frac{1}{l} \iint_{\Pi} \varphi_{x y}^{2,2} \sin \mu_{m} x \sin \mu_{n} y d x d y+ \\
+\frac{1}{\mu_{m}^{2} \mu_{n}^{2}} \frac{1}{l} \iint_{\Pi} \varphi_{x y}^{2,2} \sin \mu_{n} x \sin \mu_{m} y d x d y= \\
=\frac{1}{\mu_{m}^{2} \mu_{n}^{2}} \iint_{\Pi} \varphi_{x y}^{2,2} Z_{m n}(x, y) d x d y=\frac{l^{4}}{\pi^{4}} \frac{\varphi_{m n}^{(4)}}{m^{2} n^{2}}
\end{gathered}
$$

$$
\begin{aligned}
& F_{m n}(t)=\iint_{\Pi} F(x, y, t) Z_{m n}(x, y) d x d y= \\
& =\frac{1}{l} \int_{0}^{l} \sin \mu_{n} y d y \int_{0}^{l} F(x, y, t) \sin \mu_{m} x d x+ \\
& +\frac{1}{l} \int_{0}^{l} \sin \mu_{m} y d y \int_{0}^{l} F(x, y, t) \sin \mu_{n} x d x= \\
& =-\frac{1}{\mu_{m}^{2}} \frac{1}{l} \int_{0}^{l} \sin \mu_{n} y d y \int_{0}^{l} F_{x x}^{\prime \prime} \sin \mu_{m} x d x- \\
& -\frac{1}{\mu_{n}^{2}} \frac{1}{l} \int_{0}^{l} \sin \mu_{m} y d y \int_{0}^{l} F_{x x}^{\prime \prime} \sin \mu_{n} x d x= \\
& =-\frac{1}{\mu_{m}^{2}} \frac{1}{l} \int_{0}^{l} \sin \mu_{m} x d x \int_{0}^{l} F_{x x}^{\prime \prime} \sin \mu_{n} y d y- \\
& -\frac{1}{\mu_{n}^{2}} \frac{1}{l} \int_{0}^{l} \sin \mu_{n} x d x \int_{0}^{l} F_{x x}^{\prime \prime} \sin \mu_{m} y d y= \\
& =\frac{1}{\mu_{m}^{2} \mu_{n}^{2}} \frac{1}{l} \int_{0}^{l} \sin \mu_{m} x d x \int_{0}^{l} F_{x y}^{2,2} \sin \mu_{n} y d y+ \\
& +\frac{1}{\mu_{m}^{2} \mu_{n}^{2}} \frac{1}{l} \int_{0}^{l} \sin \mu_{n} x d x \int_{0}^{l} F_{x y}^{2,2} \sin \mu_{m} y d y= \\
& =\frac{1}{\mu_{m}^{2} \mu_{n}^{2}} \frac{1}{l} \iint_{\Pi} F_{x y}^{2,2} \sin \mu_{m} x \sin \mu_{n} y d x d y+ \\
& +\frac{1}{\mu_{m}^{2} \mu_{n}^{2}} \frac{1}{l} \iint_{\Pi} F_{x y}^{2,2} \sin \mu_{n} x \sin \mu_{m} y d x d y= \\
& =\frac{1}{\mu_{m}^{2} \mu_{n}^{2}} \iint_{\Pi} F_{x y}^{2,2} Z_{m n}(x, y) d x d y=\frac{l^{4}}{\pi^{4}} \frac{F_{m n}(t)^{(4)}}{m^{2} n^{2}} \text {. }
\end{aligned}
$$

Поскольку $\varphi_{x y}^{2,2}$ и $F_{x y}^{2,2}(t)$ непрерывны в $\bar{\Pi}$, в силу неравенства Бесселя следующие ряды сходятся:

$$
\begin{gathered}
\sum_{m, n=1}^{\infty}\left|\varphi_{m n}^{(4)}\right|^{2} \leqslant \frac{2}{l} \int_{0}^{l} \int_{0}^{l}\left(\varphi_{x y}^{2,2}\right)^{2} d x d y \\
\sum_{m, n=1}^{\infty}\left|F_{m n}^{(4)}(t)\right|^{2} \leqslant \frac{2}{l} \int_{0}^{l} \int_{0}^{l}\left(F_{x y}^{2,2}(t)\right)^{2} d x d y .
\end{gathered}
$$


Подставив (22) и (23) в ряд (21), получим

$$
u(x, y, t)=\frac{l^{4}}{\pi^{4}} \sum_{m, n=1}^{\infty} \frac{1}{m^{2} n^{2}}\left[\varphi_{m n}^{(4)} e^{-\mu_{m n}^{2} a^{2} t}+\int_{0}^{t} F_{m n}^{(4)}(s) e^{-\mu_{m n}^{2} a^{2}(t-s)} d s\right] Z_{m n}(x, y) .
$$

Теперь оценим

$$
\begin{gathered}
\left|\int_{0}^{t} F_{m n}^{(4)}(s) e^{-\mu_{m n}^{2} a^{2}(t-s)} d s\right|=\max _{0 \leqslant s \leqslant T}\left|F_{m n}^{(4)}(s)\right| \int_{0}^{t} e^{-\mu_{m n}^{2} a^{2}(t-s)} d s= \\
=\frac{\left\|F_{m n}^{(4)}\right\|_{C}\left(1-e^{-\mu_{m n}^{2} a^{2} t_{0}}\right)}{\mu_{m n}^{2} a^{2}}<\frac{l^{2}}{a^{2} \pi^{2}} \frac{\left\|F_{m n}^{(4)}\right\|_{C}}{m^{2}+n^{2}} .
\end{gathered}
$$

Ряд (24) при любых $(x, y, t) \in \bar{D}$ мажорируется сходящимся рядом

$$
\frac{l^{4}}{\pi^{4}} \sum_{m, n=1}^{\infty} \frac{1}{m^{2} n^{2}}\left|\varphi_{m n}^{(4)}\right|+\frac{l^{6}}{\pi^{6} a^{2}} \sum_{m=1}^{\infty} \frac{1}{m^{2}+n^{2}} \sum_{n=1}^{\infty} \frac{\left\|F_{m n}^{(4)}\right\|_{C}}{m^{2} n^{2}} .
$$

Тогда ряд (21) в силу признака Вейерштрасса сходится абсолютно и равномерно в $\bar{D}$. Следовательно, функция $u(x, y, t)$ непрерывна в $\bar{D}$ как сумма равномерно сходящегося ряда (21).

Теперь докажем возможность почленного дифференцирования ряда (21) по переменным $x, y$ два раза и по $t$ один раз в $D$. Для этого покажем, что полученные при почленном дифференцировании ряды сходятся абсолютно и равномерно на $\bar{D}_{0}=\bar{D} \cap\left\{t \geqslant t_{0}>0\right\}$, где $t_{0}$ - достаточно малое положительное число. Формально из (24) почленным дифференцированием составим следующие ряды:

$$
\begin{aligned}
& u_{x x}=-\frac{l^{4}}{\pi^{4}} \sum_{m, n=1}^{\infty} \frac{\mu_{m}^{2}}{m^{2} n^{2}}\left(\varphi_{m n}^{(4)} e^{-\mu_{m n}^{2} a^{2} t}+\right. \\
& \left.+\int_{0}^{t} F_{m n}^{(4)}(s) e^{-\mu_{m n}^{2} a^{2}(t-s)} d s\right) Z_{m n}(x, y), \\
& u_{y y}=-\frac{l^{4}}{\pi^{4}} \sum_{m, n=1}^{\infty} \frac{\mu_{n}^{2}}{m^{2} n^{2}}\left(\varphi_{m n}^{(4)} e^{-\mu_{m n}^{2} a^{2} t}+\right. \\
& \left.+\int_{0}^{t} F_{m n}^{(4)}(s) e^{-\mu_{m n}^{2} a^{2}(t-s)} d s\right) Z_{m n}(x, y) \\
& u_{t}=\frac{l^{4}}{\pi^{4}} \sum_{m, n=1}^{\infty} \frac{\mu_{m n}^{2} a^{2}}{m^{2} n^{2}}\left(-\varphi_{m n}^{(4)} e^{-\mu_{m n}^{2} a^{2} t}+\right. \\
& \left.+\int_{0}^{t} F_{m n}^{(4)}(s) e^{-\mu_{m n}^{2} a^{2}(t-s)} d s+\frac{F_{m n}^{(4)}(t)}{\mu_{m n}^{2} a^{2}}\right) Z_{m n}(x, y) .
\end{aligned}
$$


Ряды $(25)-(27)$ при любых $(x, y, t) \in \bar{D}_{0}$ мажорируются соответственно рядами

$$
\begin{aligned}
& \frac{l^{4}}{\pi^{4}} \sum_{m, n=1}^{\infty} \frac{1}{m^{2} n^{2}}\left|\varphi_{m n}^{(4)}\right| \mu_{m}^{2} e^{-\mu_{m n}^{2} a^{2} t}+\frac{l^{5}}{\pi^{5} a^{2}} \sum_{m=1}^{\infty} \frac{1}{m\left(m^{2}+n^{2}\right)} \sum_{n=1}^{\infty} \frac{\left\|F_{m n}^{(4)}\right\|_{C}}{n^{2}}, \\
& \frac{l^{4}}{\pi^{4}} \sum_{m, n=1}^{\infty} \frac{1}{m^{2} n^{2}}\left|\varphi_{m n}^{(4)}\right| \mu_{n}^{2} e^{-\mu_{m n}^{2} a^{2} t}+\frac{l^{5}}{\pi^{5} a^{2}} \sum_{n=1}^{\infty} \frac{1}{n\left(m^{2}+n^{2}\right)} \sum_{m=1}^{\infty} \frac{\left\|F_{m n}^{(4)}\right\|_{C}}{m^{2}} \\
& \frac{l^{4} a^{2}}{\pi^{4}} \sum_{m, n=1}^{\infty} \frac{1}{m^{2} n^{2}}\left|\varphi_{m n}^{(4)}\right| \mu_{m n}^{2} e^{-\mu_{m n}^{2} a^{2} t}+\frac{2 l^{4}}{\pi^{4}} \sum_{m=1}^{\infty} \frac{1}{m^{2}} \sum_{n=1}^{\infty} \frac{\left\|F_{m n}^{(4)}\right\|_{C}}{n^{2}}
\end{aligned}
$$

Ряды (28)-(30) сходятся абсолютно. Тогда ряды (25)-(27) на основании признака Вейерштрасса сходятся абсолютно и равномерно на $D$. Следовательно, функции $u_{x x}, u_{y y}, u_{t} \in C(D)$ и, подставляя ряды в уравнение $(21)$, убеждаемся в том, что функция $u(x, y, t)$, определяемая рядом $(21)$, является его решением в $D$.

\section{3. Критерий единственности решения обратной задачи}

Рассмотрим теперь обратную задачу (2)-(7). Полагая в формуле (21) $x=x_{0}, y=y_{0}$ с учётом условия $(7)$, получим относительно неизвестной функции $\varphi(x, y)$ уравнение

$$
\begin{aligned}
& \sum_{m, n=1}^{\infty} \iint_{\Pi} \varphi(\xi, \eta) Z_{m n}(\xi, \eta) d \xi d \eta e^{-\mu_{m n}^{2} a^{2} t} Z_{m n}\left(x_{0}, y_{0}\right)= \\
&=h(t)-\sum_{m, n=1}^{\infty} F_{m n}(t) Z_{m n}\left(x_{0}, y_{0}\right)=h_{0}(t), \quad t_{0} \leqslant t \leqslant t_{1}
\end{aligned}
$$

Теорема 3. Если $Z_{m n}\left(x_{0}, y_{0}\right) \neq 0$ при всех $m, n \in \mathbb{N}$, то решение интегрального уравнения (31) единственно в $L_{2}(\bar{\Pi})$.

Доказ а тель ст во. Следуя [1, с. 119], [7,9], в силу линейности уравнения (31) достаточно показать, что оно имеет только нулевое решение при $h_{0}(t)=0$. Положив в $(31) h(t)=0$ и $F(x, y, t) \equiv 0$, имеем

$$
\sum_{m, n=1}^{\infty} \iint_{\Pi} \varphi(\xi, \eta) Z_{m n}(\xi, \eta) d \xi d \eta e^{-\mu_{m n}^{2} a^{2} t} Z_{m n}\left(x_{0}, y_{0}\right)=0
$$

Рассмотрим в комплексной полуплоскости $\operatorname{Re} z \geqslant \alpha$, где $\alpha \in\left(0, t_{0}\right)$ - постоянная, функцию комплексной переменной

$$
\Phi(z)=\sum_{m, n=1}^{\infty} \varphi_{m n} e^{-\mu_{m n}^{2} a^{2} z} Z_{m n}\left(x_{0}, y_{0}\right)
$$

Так как при $\operatorname{Re} z \geqslant \alpha$

$$
\left|\varphi_{m n} Z_{m n}\left(x_{0}, y_{0}\right) e^{-\mu_{m n}^{2} a^{2} z}\right| \leqslant C e^{-\mu_{m n}^{2} a^{2} \alpha},
$$


где $C=$ const $>0$, то в этой полуплоскости ряд, стоящий в правой части соотношения (33), сходится равномерно. Учитывая, что каждый член этого ряда является аналитической функцией при $\operatorname{Re} z \geqslant \alpha$, и применяя теорему Вейерштрасса, получаем, что функция $\Phi(z)$ является аналитической при $\operatorname{Re} z \geqslant \alpha$. Поскольку в силу $(32) \Phi(z)=0$ на отрезке $\left[t_{0}, t_{1}\right]$ действительной оси $t$ из области аналитичности $\Phi(z)$, то на основании теоремы единственности для аналитических функций следует $\Phi(z) \equiv 0$ при $\operatorname{Re} z \geqslant \alpha$. Отсюда следует равенство при всех $t \geqslant t_{0}$ :

$$
\sum_{m, n=1}^{\infty} \varphi_{m n} e^{-\mu_{m n}^{2} a^{2} t} Z_{m n}\left(x_{0}, y_{0}\right)=0 .
$$

Умножим равенство $(34)$ на $e^{\left(\mu_{11} a\right)^{2} t}$ и в полученном равенстве, переходя к пределу при $t \rightarrow+\infty$, найдем

$$
\varphi_{11}=0
$$

С учетом (35) умножим равенство (34) на $e^{\left(\mu_{12} a\right)^{2} t}$ и в полученном равенстве, переходя к пределу при $t \rightarrow+\infty$, получим

$$
\varphi_{12} Z_{12}\left(x_{0}, y_{0}\right)+\varphi_{21} Z_{21}\left(x_{0}, y_{0}\right)=2 \varphi_{12} Z_{12}\left(x_{0}, y_{0}\right)=0 \text {. }
$$

Отсюда с учетом того, что $Z_{12}\left(x_{0}, y_{0}\right) \neq 0$, имеем

$$
\varphi_{12}=\varphi_{21}=0
$$

Снова учитывая (35) и (36), умножая равенство (34) на $e^{\left(\mu_{22} a\right)^{2} t}$ и переходя к пределу при $t \rightarrow+\infty$ в полученном равенстве, найдем

$$
\varphi_{22}=0
$$

Затем, учитывая равенства (35)-(37), умножим равенство (34) на $e^{\left(\mu_{13} a\right)^{2} t}$ и, переходя к пределу при $t \rightarrow \infty$, будем иметь

$$
\varphi_{13}=\varphi_{31}=0 \text {. }
$$

Затем в силу (35)-(38) получим

$$
\varphi_{23}=\varphi_{32}=0 \text {. }
$$

Рассуждая далее аналогично вышеизложенному, получим

$$
\varphi_{m n}=0, \quad m, n=1,2, \ldots
$$

Из равенства (39) в силу полноты системы функций $\left\{Z_{m n}(x, y)\right\}_{m, n \geqslant 1}$ в пространстве $L_{2}(\bar{\Pi})$ следует, что $\varphi(x, y)=0$ почти всюду в П. Отсюда в силу непрерывности функции $\varphi(x, y)$ на $\bar{\Pi}$ получим, что $\varphi(x, y) \equiv 0$.

Тогда из теорем 3 и 2 следует единственность решения обратной задачи $(2)-(7)$. 
Пусть при некоторых $\widetilde{x}_{0}=x_{0} / l, \widetilde{y}_{0}=y_{0} / l$ и $m=p, n=s \in \mathbb{N}$ нарушено условие теоремы 3 :

$$
Z_{p s}\left(x_{0}, y_{0}\right)=\sin \pi p \widetilde{x}_{0} \sin \pi s \widetilde{y}_{0}+\sin \pi s \widetilde{x}_{0} \sin \pi p \widetilde{y}_{0}=0
$$

Тогда обратная задача $(2)-(7)$ при $h(t)=0$ и $F(x, y, t) \equiv 0$ имеет ненулевое решение

$$
u_{p s}(x, y, t)=Z_{p s}(x, y) e^{-\left(\mu_{p s} a\right)^{2} t}, \quad \varphi_{p s}(x, y)=u_{p s}(x, y, 0)=Z_{p s}(x, y) .
$$

Из уравнения (40) найдем рациональные значения

$$
\widetilde{x}_{0}=k_{1} / p, \quad \widetilde{y}_{0}=k_{2} / p, \quad k_{1}, k_{2} \in \mathbb{N}, \quad k_{1}<p, k_{2}<p,
$$

или

$$
\widetilde{x}_{0}=k_{3} / s, \quad \widetilde{y}_{0}=k_{4} / s, \quad k_{3}, k_{4} \in \mathbb{N}, \quad k_{3}<s, k_{4}<s
$$

при которых нарушается условие теоремы 3, т.е. нарушается единственность решения задачи (2)-(7).

Следовательно, установлен следующий критерий единственности решения обратной задачи (2)-(7).

Теорема 4. Условия $Z_{m n}\left(x_{0}, y_{0}\right) \neq 0$ nри всех $m, n \in \mathbb{N}$ необходимы и достаточны для единственности решения обратной задачи (2)-(7).

Рассматриваемая обратная задача может быть сведена к интегральному уравнению Фредгольма первого рода. Действительно, поменяв местами порядок интегрирования и суммирования в левой части уравнения (31), получим интегральное уравнение Фредгольма первого рода

$$
G \varphi \equiv \iint_{\Pi} G(t, \xi, \eta) \varphi(\xi, \eta) d \xi d \eta=h_{0}(t), t_{0} \leqslant t \leqslant t_{1},
$$

с гладким ядром

$$
G(t, \xi, \eta)=\sum_{m, n=1}^{\infty} e^{-\mu_{m n}^{2} a^{2} t} Z_{m n}(\xi, \eta) Z_{m n}\left(x_{0}, y_{0}\right)
$$

в параллелепипеде $\bar{\Pi} \times\left[t_{0}, t_{1}\right]$. Следовательно, интегральный оператор $G$, pacсматриваемый действующим из $L_{2}(\bar{\Pi})$ в $L_{2}\left[t_{0}, t_{1}\right]$, вполне непрерывен. Задача решения уравнения (41) в этой паре пространств некорректна.

\section{4. Заключение}

В заключение отметим, что обратные задачи для уравнений параболического типа, как правило, являются некорректными. Для их решения обычно привлекается теория некорректных задач, разработанная в работах А. Н. Тихонова, М. М. Лаврентьева, В. К. Иванова и их учеников [10-12]. В данной работе, в отличие от других работ, предлагается спектральный метод для обоснования единственности решения обратной задачи (2)-(7).

Благодарности. Работа выполнена при поддержке Российского фонда фундаментальных исследований (проект № 14-01-97003-р_поволжье_а).

\section{ORCID}

Артур Рашитович Зайнуллов: http://orcid.org/0000-0003-2657-4101 


\section{БИБЛИОГРАФИЧЕСКИЙ СПИСОК}

1. Денисов А. М. Введение в теорию обратных задач. М.: МГУ, 1994. 208 с.

2. Тихонов А. Н., Самарский А. Н. Уравнения математической физики. М.: Физматлит, 1966. $724 \mathrm{c}$.

3. Соболев С. Л. Уравнения математической физики. М.: Наука, 1992. 432 с.

4. Владимиров В. С., Жаринов В. В. Уравнения математической физики. М.: Физматлит, 2000. 398 с.

5. Кошляков Н. С., Глинер Э. Б., Смирнов М. М. Уравнения в частных производных математической физики. М.: Высшая школа, 1970. 712 с.

6. Годунов С. К. Уравнения математической физики. М.: Наука, 1978. 392 с.

7. Сабитов К. Б. Уравнения математической физики. М.: Физматлит, 2013. 352 с.

8. Сабитов К. Б. Об одной краевой задаче для уравнения смешанного типа третьего порядка // Докл. РАН, 2009. Т. 427, № 5. С. 593-596.

9. Зайнуллов А. Р. Обратные задачи для уравнения теплопроводности // Вестн. СамГУ. Естественнонаучн. сер., 2015. №6(128). С. 62-75.

10. Тихонов А. Н., Арсенин В. Я. Методъ решения некорректных задач. М.: Наука, 1979. 285 c.

11. Лавретьев М. М., Романов В. Г., Шишатский С. П. Некорректные задачи математической физики и анализа. М.: Наука, 1980. 286 с.

12. Иванов В. К., Васин В. В., Танана В. П. Теория линейных некорректных задач и её приложения. М.: Наука, 1978. 206 с.

Поступила в редакцию $10 / \mathrm{X} / 2015$;

в окончательном варианте - $12 / \mathrm{XI} / 2015$;

принята в печать - 19/XI/2015. 
Vestn. Samar. Gos. Techn. Un-ta. Ser. Fiz.-mat. nauki

[J. Samara State Tech. Univ., Ser. Phys. \& Math. Sci.], 2015, vol. 19, no. 4, pp.667-679

ISSN: 2310-7081 (online), 1991-8615 (print)

doi: http://dx.doi.org/10.14498/vsgtu1451

MSC: 35G16, 35R30

\title{
AN INVERSE PROBLEM FOR TWO-DIMENSIONAL EQUATIONS OF FINDING THE THERMAL CONDUCTIVITY OF THE INITIAL DISTRIBUTION
}

\author{
A. R. Zaynullov \\ Sterlitamak Branch of Bashkir State University, \\ 49, Lenin Avenue, Sterlitamak, 453103, Russian Federation.
}

\begin{abstract}
The inverse problem of finding the initial distribution has been studied on the basis of formulas for the solution of the first initial-boundary value problem for the inhomogeneous two-dimentional heat equation. The uniqueness of the solution of the direct initial-boundary value problem has proved with the completeness of the eigenfunctions of the corresponding homogeneous Dirichlet problem for the Laplace operator. The existence theorem for solving direct initial boundary value problem has been proved. Inverse problem has been investigated on the basis of the solution of direct problem, a criterion for the uniqueness of the inverse problem of finding the initial distribution has been proved. The existence of the inverse problem solution has been equivalently reduced to Fredholm integral equation of the first kind.
\end{abstract}

Keywords: heat equation, first initial-boundary value problem, inverse problem, spectral method, uniqueness, existence, integral equation.

doi: http://dx.doi.org/10.14498/vsgtu1451

Acknowledgments. This work was supported by the Russian Foundation for Basic Research (project no. 14-01-97003-r_povolzh'e_a).

\section{ORCID}

Artur R. Zaynullov: http: //orcid.org/0000-0003-2657-4101

\section{REFERENCES}

1. Denisov A. M. Elements of the theory of inverse problems, Inverse and Ill-Posed Problems Series. Utrecht, VSP, 1999. iv+272 pp. doi: 10.1515/9783110943252.

2. Tikhonov A. N.; Samarskii A. A. Equations of mathematical physics, International Series of Monographs on Pure and Applied Mathematics, vol. 39. Oxford etc., Pergamon Press, 1963, xvi+765 pp.

(C) 2015 Samara State Technical University.

Please cite this article in press as:

Zaynullov A. R. An inverse problem for two-dimensional equations of finding the thermal conductivity of the initial distribution, Vestn. Samar. Gos. Tekhn. Univ., Ser. Fiz.-Mat. Nauki [J. Samara State Tech. Univ., Ser. Phys. \& Math. Sci.], 2015, vol. 19, no. 4, pp. 667-679. doi: 10.14498/vsgtu1451. (In Russian)

Author Details:

Artur R. Zaynullov (arturzayn@mail.ru), Postgraduate Student, Dept. of Mathematical Analysis. 
3. Sobolev S. L. Uravneniia matematicheskoi fiziki [Equations of mathematical physics]. Moscow, Nauka, 1992, 432 pp. (In Russian)

4. Vladimirov V. S., Zharinov V. V. Uravneniia matematicheskoi fiziki [Equations of mathematical physics]. M., Fizmatlit, 2000, 398 pp. (In Russian)

5. Koshlyakov N. S., Smirnov M. M., Gliner E. B. Differential equations of mathematical physics. Amsterdam, North-Holland Publishing Company, 1964, xvi+701 pp.

6. Godunov S. K. Uravneniia matematicheskoi fiziki [Equations of mathematical physics]. Moscow, Nauka, 1978, 392 pp. (In Russian)

7. Sabitov K. B. Uravneniia matematicheskoi fiziki [Equations of mathematical physics]. Moscow, Fizmatlit, 2013, 352 pp. (In Russian)

8. Sabitov K. B. A boundary value problem for a third-order equation of mixed type, Dokl. Math., 2009, vol. 80, no. 1, pp. 565-568. doi: 10.1134/S1064562409040292.

9. Zaynullov A. R. Inverse problems for the heat equation, Vestnik SamGU. EstestvennoNauchnaya Ser., 2015, no.6(128), pp. 62-75 (In Russian).

10. Tikhonov A. N., Arsenin V. Ya. Solutions of ill-posed problems, Scripta Series in Mathematics. New York etc., John Wiley \& Sons, 1977, xiii+258 pp.

11. Lavrent'ev M. M., Romanov V. G., Shishatskij S. P. Ill-posed problems of mathematical physics and analysis, Translations of Mathematical Monographs, vol.64. Providence, R.I., American Mathematical Society, 1986, vi+290 pp.

12. Ivanov V. K., Vasin V. V., Tanana V. P. Theory of linear ill-posed problems and its applications, Inverse and Ill-Posed Problems Series. Utrecht, VSP, 2002 xiii+281 pp. doi : 10.1515/9783110944822.

Поступила в редакцию $10 / \mathrm{X} / 2015$; в окончательном варианте - $12 / \mathrm{XI} / 2015$; принята в печать - 19/XI/2015. 